\title{
BOUNDS FOR ZEROS OF SOME SPECIAL FUNCTIONS
}

\author{
HERBERT W. HETHCOTE ${ }^{1}$
}

\begin{abstract}
For $n \geqq 1$ let $b_{n}$ and $c_{n}$ be zeros (ordered by increasing values) of $u(x)$ and $v(x)$, respectively, which are nontrivial solutions of $u^{\prime \prime}+p(x) u=0$ and $v^{\prime \prime}+q(x) v=0$ with continuous $p(x)$ and $q(x)$. It is shown that if $b_{n}-c_{n} \rightarrow 0$ as $n \rightarrow \infty, p(x) \geqq$ $q(x)$, and either $p(x)$ or $q(x)$ is nonincreasing, then $b_{n} \geqq c_{n}$ for $n \geqq 1$. Inequalities related to asymptotic expansions are obtained for the negative zeros $a_{n}$ of the Airy function $A i(z)$ and the zeros $j_{\nu, n}$ of the Bessel function $J_{\nu}(x)$.
\end{abstract}

The principal theorem, which gives an inequality for zeros of solutions of linear second order differential equations, is proved by induction using the Sturm comparison theorem at each step. This procedure differs from previous applications of the Sturm comparison theorem to orthogonal polynomials [3, pp. 120-130] and Bessel functions [4, pp. 518-521] since the common zero of the solutions of the differential equations is at infinity here, i.e., approached asymptotically.

TheOREM 1. For $n \geqq 1$ let $b_{n}$ and $c_{n}$ be zeros (ordered by increasing values) of $u(x)$ and $v(x)$, respectively, which are nontrivial solutions of $u^{\prime \prime}+p(x) u=0$ and $v^{\prime \prime}+q(x) v=0$ with continuous $p(x)$ and $q(x)$. If $b_{n}-c_{n} \rightarrow 0$ as $n \rightarrow \infty, p(x) \geqq q(x)$, and either $p(x)$ or $q(x)$ is nonincreasing, then $b_{n} \geqq c_{n}$ for $n \geqq 1$.

Proof. The zeros $b_{n}$ and $c_{n}$ are simple and cannot have a finite accumulation point [1, pp. 223-225]. For $p(x) \equiv q(x)$, the theorem is true since $b_{n}=c_{n}$. Assume $p(x) \not \equiv q(x)$ and $b_{n}<c_{n}$ for some $n$. Let $k$ be the least positive integer such that $b_{k}<c_{k}$. If we let $d_{n}=c_{n}-b_{n}$, then $d_{k}>0$. We will show by induction that $d_{n} \geqq d_{k}>0$ for all $n \geqq k$. Assume $d_{m} \geqq d_{k}>0$ for some $m \geqq k$. If $w(x)=v\left(x+d_{m}\right)$, then $w^{\prime \prime}(x)+q\left(x+d_{m}\right) w(x)$ $=0, w\left(b_{m}\right)=0$, and $w\left(c_{m+1}-d_{m}\right)=0$. Since either $p(x) \geqq p\left(x+d_{m}\right)$ $\geqq q\left(x+d_{m}\right)$ or $p(x) \geqq q(x) \geqq q\left(x+d_{m}\right)$, the Sturm comparison theorem implies $b_{m}<b_{m+1}<c_{m+1}-d_{m}$. Thus $c_{m+1}-b_{m+1}=d_{m+1}>d_{m} \geqq d_{k}>0$, which completes our induction proof. However, $c_{n}-b_{n}=d_{n} \geqq d_{k}>0$ for all $n \geqq k$ contradicts $b_{n}-c_{n} \rightarrow 0$. Consequently, no least positive

Received by the editors August 18, 1969.

A MS Subject Classifications. Primary 3442, 4150, 3325; Secondary 3450.

Key Words and Phrases. Zeros of special functions, asymptotic expansions, approximation of zeros, Sturm comparison theorem, Airy functions, Bessel functions.

1 This research was supported by Project Themis, under Contract No. DAAF0369-C-0014. 
integer $k$ exists such that $b_{k}<c_{k}$ so that we must have $b_{n} \geqq c_{n}$ for all positive integers $n$.

THEOREM 2. If $a_{n}$ is the nth negative zero of the Airy function Ai(z), then for $n \geqq 1$,

$-\left[\frac{3 \pi}{8}(4 n-1)+\frac{3}{2} \arctan \frac{5}{18 \pi(4 n-1)}\right]^{2 / 3} \leqq a_{n} \leqq-\left[\frac{3 \pi}{8}(4 n-1)\right]^{2 / 3}$.

Proof. It is well known $\left[2\right.$, p. B48] that $a_{n}+[3 \pi(4 n-1) / 8]^{2 / 3} \rightarrow 0$ as $n \rightarrow \infty$. The function $u(\zeta)=\pi^{1 / 2} x^{1 / 4} A i(-x)$ where $\zeta=2 x^{3 / 2} / 3$ satisfies $u^{\prime \prime}+\left(1+5 /\left(36 \zeta^{2}\right)\right) u=0$ and $v(\zeta)=\cos (\zeta-\pi / 4)$ satisfies $v^{\prime \prime}+v=0$. From Theorem 1 we have $2\left(-a_{n}\right)^{3 / 2} / 3 \geqq n \pi-\pi / 4$ from which the right-hand inequality follows immediately.

To prove the left-hand inequality we let

$$
u(\zeta)=\frac{\left[(72)^{2} \zeta^{2}+25\right]^{1 / 2}}{\left[(72)^{2} \zeta^{2}+385\right]^{1 / 2}} \cos \left(\zeta-\pi / 4-\arctan \frac{5}{72 \zeta}\right)
$$

which satisfies

$$
u^{\prime \prime}+\left(1+\frac{5}{36 \zeta^{2}}+\frac{385\left[206(72)^{2} \zeta^{2}-3850\right]}{72 \zeta^{2}\left[(72)^{2} \zeta^{2}+385\right]^{2}}\right) u=0 .
$$

If we now apply Theorem 1 with $v(\zeta)=\pi^{1 / 2} x^{1 / 4} A i(-x)$, then the already established inequality implies 206(72) ${ }^{2} \zeta^{2}>3850$. Consequently, $2\left(-a_{n}\right)^{3 / 2} / 3 \leqq b_{n}$ where

$$
b_{n}-\pi / 4-\arctan \frac{5}{72 b_{n}}=n \pi-\pi / 2 .
$$

The left-hand inequality now follows from

$$
b_{n} \leqq n \pi-\pi / 4+\arctan (5 / 18 \pi(4 n-1)) .
$$

TheоRem 3. If $j_{v, n}$ is the nth positive zero of the Bessel function $J_{\nu}(x)$ for $\nu \geqq 0$, then for $\nu \leqq 1 / 2$,

$$
n \pi+\frac{\nu \pi}{2}-\frac{\pi}{4} \leqq j_{\nu, n} \leqq n \pi+\frac{\nu \pi}{2}-\frac{\pi}{4}-\frac{4 \nu^{2}-1}{8\left(n \pi+\frac{\nu \pi}{2}-\frac{\pi}{4}\right)} ;
$$

and for $\nu \geqq 1 / 2, j_{v, n} \leqq n \pi+\nu \pi / 2-\pi / 4$.

Proof. The inequalities in the above theorem are related to McMahon's asymptotic expansion of $j_{r, n}[4$, p. 506]. The proof follows from Theorem 1 when the following functions and dif- 
ferential equations are considered with $\alpha=\left(4 \nu^{2}-1\right) / 8$. The function $(\pi x / 2)^{1 / 2} J_{\nu}(x)$ satisfies $u^{\prime \prime}+\left(1-2 \alpha / x^{2}\right) u=0$, and $\cos (x-\nu \pi / 2-\pi / 4)$ satisfies $v^{\prime \prime}+v=0$. The function

$$
\left(1-\alpha / x^{2}\right)^{-1 / 2} \cos (x-\nu \pi / 2-\pi / 4+\alpha / x)
$$

satisfies

$$
w^{\prime \prime}+\left(1-\frac{2 \alpha}{x^{2}}+\frac{\alpha^{2}\left(x^{2}-\alpha\right)^{2}-3 \alpha x^{4}}{x^{4}\left(x^{2}-\alpha\right)^{2}}\right) w=0 .
$$

\section{REFERENCES}

1. E. L. Ince, Ordinary differential equations, reprint, Dover, New York, 1944. MR 6, 65.

2. J. C. P. Miller, The Airy integral, giving tables of solutions of the differential equation $y^{\prime \prime}=x y$, British Assoc. for the Advancement of Science Math. Tables, partvol. B, Cambridge Univ. Press, New York, 1946. MR 8, 353.

3. G. Szegö, Orthogonal polynomials, Amer. Math. Soc. Colloq. Publ., vol. 23, Amer. Math. Soc., Providence, R. I., 1939. MR 1, 14.

4. G. N. Watson, A treatise on the theory of Bessel functions, 2nd ed., Cambridge Univ. Press, Cambridge, 1944. MR 6, 64.

University of Iowa, Iowa City, Iowa 52240 